\title{
Percepciones sobre el ejercicio del rol directivo y sus alcances para el aprendizaje de la democracia
}

\author{
Perceptions of the exercise of managerial role and scope for learning democracy
}

Recibido 17 setiembre 2015 • Aceptado 06 octubre 2015 • Corregido 10 noviembre 2015

\author{
Carolina Aparicio Molina ${ }^{1}$ \\ Departamento de Fundamentos de la Pedagogía \\ Universidad Católica de la Santísima Concepción \\ Concepción, Chile \\ caparicio@ucsc.cl
}

\begin{abstract}
Resumen. A la luz de las teorías actuales sobre dirección y liderazgo escolar, en este artículo se analizan las perspectivas de directivos en ejercicio respecto a su rol en la formación democrática y para la participación de los estudiantes en institutos de secundaria de dos centros educativos de Chile y dos de España. El análisis provino de entrevistas en profundidad a los cuatro directivos, los cuales situaron el ejercicio de su rol en los ejes de formación para el diálogo y la convivencia, específicamente para desarrollar un aprendizaje vital validado desde sus creencias personales. Junto con analizar las percepciones reveladas, el artículo busca poner en discusión la necesaria revisión de los roles ejercidos por las personas líderes escolares en materia de formación ciudadana, a fin de promover el diálogo sobre su importancia para la puesta en práctica de experiencias integrales de democracia junto con la comunidad educativa.
\end{abstract}

Palabras claves. Gestión educacional; educación ciudadana; democratización de la educación

Abstract. In light of principal theories about school leadership and management, in this paper we analyzed the views of principals about their role in the education for democratic and social participation at Chilean and Spain secondary institutes. We analyze interviews of four principals from two institutes in Chile and two in Spain, they said that their role was close from dialogue and school climate and learning from vital experiences.

Despite the results, this paper aims discuss the role of principals about the importance of teaching and learning citizenship, generate dialogue at schools and increase the creation of democratic spaces for the management with the scholar community.

Keywords. Educational management; civic education; democratization of education

1 Doctora en Educación y Sociedad de la Universidad de Barcelona. Magíster en Desarrollo Humano y Licenciada en Educación de la Universidad Austral de Chile. Actualmente se desempeña como académica en la Universidad Católica de la Santísima Concepción (Chile). 


\section{Gestión y liderazgo en las escuelas del Siglo XXI}

La perspectiva de la gestión escolar que comprende, valora y acciona un directivo, puede hacer que las prácticas instauradas en la escuela sean una profunda experiencia de aprendizaje de y para la vida en sociedad (Murillo, 2006). Especialmente, considerando que el aprendizaje de la participación social y de la democracia como práctica organizacional se vincula estrechamente con el modo de gestión y de convivencia que pueden vivir los estudiantes dentro de una escuela (Puig, Martin y Trilla, 1998). Lo que, entre otras cosas, ha demostrado mejorar aspectos fundamentales de la vida en la escuela como la inclusión, limitando con ello, las barreras para que todos los estudiantes desarrollen aprendizajes de calidad, situación altamente valorada en la sociedad actual (Booth \& Ainscow, 2002).

En esta línea, durante los últimos años se han reportado múltiples estudios que dotan de una particular relevancia a las características de la dirección escolar; entre ellos, se encuentran la importancia de un liderazgo tendiente a la democratización de la gestión, como lo han indicado desde diferentes perspectivas autores como Fullan \& Hargreaves (1999); Murillo (2006); Harris \& Chapman (2002); Bolívar, López \& Murillo (2013) y otros. Sin embargo, y a pesar del destacado papel que tiene el director de escuela, se ha situado la necesidad de pasar de la individualización del liderazgo escolar a una visión colaborativa (Riveros-Barrera, 2012), en la cual el líder se centre en aspecto esenciales para el funcionamiento de la institución pero genere espacios para que los demás también asuman responsabilidades que permitan, entre otras cosas, una adecuada interacción y funcionamiento de las redes dentro y fuera de la escuela (Fullan, 2001; Harris, 2012).

Incluso considerando esta tendencia, sigue siendo frecuente la revisión a las características personales de los líderes de las escuelas, para lo cual se ha generado una amplia caracterización de valores, competencias y actitudes deseables en quienes ejercen esos cargos. En esta línea, Goleman (2002) ha indicado que si bien el liderazgo se encontraría junto a un amplio margen de competencias, es importante la individualización de estas para promover, especialmente, la reflexión y la transformación de los directores, según las necesidades de sus escuelas. En consecuencia, entre los aspectos destacados de la caracterización de los directivos y líderes escolares, se encuentran asuntos siempre controvertidos como el carisma y la vocación de servicio; la valorización a la democracia; la creatividad, especialmente en la resolución de problemas; la facilidad para trabajar bajo supuestos de cambio y una adecuada inteligencia emocional, como lo han retratado Lorenzo (2005) y Fullan (2001), entre muchos otros.

Las características anteriormente mencionadas, se relacionan, a su vez, con una creciente tendencia a discutir, revisar y replantear el rol de la escuela en la formación ciudadana en sociedades más complejas, donde predomine la democracia y el bien común. Bajo esta premisa, es importante definir y analizar el concepto de democracia utilizado en el texto.

La democracia, desde la cual se entiende la formación ciudadana y los aportes de los 
directivos en esta materia, se corresponden a una visión de la organización de la vida en común que considera diversos aspectos, los cuales, según Beetham \& Boyle (1996), tienen que ver con la forma de tomar decisiones, de organizar la vida social y que conlleva significados, valores y principios universales. Entre esos valores y principios universales se encuentra la participación de los individuos, aspecto que, como Arnstein (1969) identificó hace décadas, involucra a lo menos cinco grados, los cuales transitan entre la manipulación de los ciudadanos por parte de quienes tienen un poder real, hasta el control de los propios ciudadanos de la toma de decisiones. Los grados varían, esencialmente, en que ciertos grados de participación no llegarían a incidir finalmente en la redistribución del poder.

Este análisis se relaciona con las críticas posteriores a la democracia formal, especialmente de la mano de la globalización tecnológica, occidentalización del desarrollo y sobrevaloración del crecimiento económico (Habermas, 2000; Klein, 2007; Beck, 2008), donde se observó "un alejamiento del ciudadano de los asuntos colectivos" (Chomsky, 2010, p. 160), la desvinculación de las comunidades y el aislamiento del sujeto, como han explicado Morin (2011) y Touraine (2005), en sus diversas obras. En consecuencia, a través de la educación de la sociedad se ha promovido una mayor participación de los ciudadanos en los asuntos que les afectan e interesan, es decir, un ejercicio directo de la democracia (Held, 1997; Habermas, 1999; Touraine, 2005; Camps, 2010). De este modo, se entiende el anhelo de un ejercicio de la democracia deliberativa, según lo planteado por Habermas (1999), en donde los ciudadanos discutan, reflexionen y lleguen a consensos respecto a las decisiones que les afectan, proceso que fortalece la sociedad civil. Este enfoque es de suma relevancia y de un prominente carácter educativo, por cuanto considera al sujeto y su capacidad de dialogar, razonar y buscar el consenso con los demás, como uno de los pilares de la democracia y de su transformación. En otras palabras, valores y actitudes como la autonomía, la capacidad de deliberar y participar efectivamente (Cogan \& Derricott, 2000), aspectos que se pueden aprender en las instituciones educativas cuando sus liderazgos consideran la relevancia de su cargo para promover esas capacidades.

Específicamente en esta materia, se espera que las próximas generaciones tengan una profunda formación en asuntos cívicos (Shulz, Ainley, Fraillon, Kerr \& Losito, 2009), que les permita integrarse activamente a la vida en democracia. Lo que sin duda, lleva a preguntarse por otros tópicos, cada vez más relevantes, para las dinámicas democráticas como son la relación entre seguidores y líderes, díada que ya han revisado destacados autores, en tanto la relación líder-seguidor necesita de un líder adecuado pero también de seguidores auténticos, dinámicos, comprometidos, etc. (Riggio, Chaleff \& Lipman- Blumen, 2008).

De esta manera, tras décadas de búsqueda y comprensión de paradigmas sobre dirección escolar, actualmente el concepto comprende tanto la preocupación por el proceso de enseñanza-aprendizaje como la visión de un liderazgo situado en una sociedad compleja y en permanente cambio. Aspecto que, como ha expresado Fullan (2001), demanda integración 
y comprensión de las distintas prácticas que convergen en la vida de la escuela más que la sola administración burocrática. Especial atención asume, desde esta óptica, la perspectiva de que las organizaciones al estar compuestas por personas, son entes vivos que aprenden y deben buscar mejorar con todos sus miembros en y con el contexto (Senge, 2005; Senge, 2012). Es decir, las organizaciones deben relacionarse activamente con su entorno, por lo cual las personas que están a favor de su funcionamiento deben buscar modos de vincularse entre ellos e impulsar la labor desde lógicas más dinámicas que la dependencia de un y único líder que administra tanto los roles como las responsabilidades de los demás.

Bajo esta revisión, surge el interés por conocer a través de la entrevista en profundidad, técnica validada para recoger y ahondar en percepciones personales (Bisquerra, 2004), la perspectiva de directivos de países de habla hispana que se encuentran, como muchos países del mundo, imbuidos en épocas de cambios y reformas educativas, a fin de alcanzar mejores aprendizajes y gestión de sus escuelas (Harris, 2012). Así, los países donde se desarrolló la investigación fueron Chile y España, específicamente en la Región del Bío-Bío del país latinoamericano y la Comunidad Autónoma de Cataluña, en España.

En cada país se entrevistó a dos directivos de institutos de secundaria, para comprender sus principales énfasis respecto a la formación democrática de los estudiantes y los alcances desde su propio ejercicio del liderazgo. El objetivo de estas entrevistas era conocer qué hacían ellos para promover la participación y desarrollo de competencias democráticas en la vida del instituto, en particular respecto a los estudiantes. Además de conocer los alcances que tenía, desde su perspectiva, la gestión y liderazgo escolar sobre los aprendizajes de la vida en democracia para los estudiantes.

El proceso de obtención de información, así como también la experiencia de compartir con directivos escolares sus propias reflexiones sobre el ejercicio de un rol tan relevante para la vida en la escuela, fue una importante fuente de aprendizaje, lo cual permitió la identificación de las principales proyecciones de estas líneas de investigación en los países latinoamericanos que demandan una revisión de la gestión escolar y el ejercicio de liderazgos que avancen hacia la democratización de la experiencia escolar.

\section{Contexto del estudio}

Es importante comprender las características del liderazgo escolar, en el marco de una sociedad que cumple con las particularidades y anhelos de una democracia del siglo XXI, retratada anteriormente, que busca ser informada, autónoma y activa respecto a los asuntos que le interesan. Elementos que, como lo ha planteado Morin (2011), significa, en consecuencia, la democratización de las diferentes instituciones que la organizan, exige la revisión en la práctica y la comprensión de los contextos donde se sitúan los institutos. En este caso, obedece a dos países hispanoparlantes, de historias similares en lo que respecta a la restauración de la escuela en períodos posteriores a dictaduras, además de un creciente interés por alcanzar mejoras en 
los resultados académicos obtenidos en pruebas estandarizadas internacionales.

En el caso de Chile, país que ha venido desarrollando, en los últimos años, una seguidilla de restructuraciones a su sistema educativo y especialmente, en los tiempos modernos, en respuesta a las demandas de la propia ciudadanía (Bellei, Contreras \& Valenzuela, 2009), ha habido una tendencia al fortalecimiento de los directivos a través del Plan de Formación de Directores. Este Plan promueve la educación permanente de los directivos, a la vez que acompaña al documento de orientaciones denominado Marco para la Buena Dirección, que aborda el quehacer directivo en distintos ámbitos de la gestión escolar. Ambos elementos se suman al Sistema de Aseguramiento de la Calidad de la Gestión Escolar, que se caracteriza por determinar las redes de colaboración y tareas que, cada institución del Sistema Educativo, debe cumplir para velar por una gestión adecuada (Tapia-Gutiérrez, Becerra-Peña, MansillaSepúlveda \& Saavedra-Muñoz, 2011; Navarro, 2007).

Respecto a la generación de instancias de gestión democrática en la escuela, las últimas reformas a la normativa chilena, como la Ley 20.370 General de Educación, publicada el 2011, retomó principios democráticos a través de un fortalecimiento de los Consejos Escolares que ya habían sido promulgados en el año 2005, por medio de la Ley 19.979. Estos Consejos son de carácter obligatorio para los establecimientos educativos que reciben fondos públicos, deben conformarse por miembros representantes de toda la comunidad escolar y cumplir con la finalidad de informar del estado del establecimiento en sus distintas áreas. Los Consejos Escolares han sido uno de los principales alicientes a la democratización de la vida en la escuela, aunque aún no se ha generado suficiente información sobre el funcionamiento de estas instancias a nivel nacional y por ende, su impacto en la formación democrática de los miembros de la comunidad escolar (Muñoz, 2011). En países como España, sí se ha registrado la relevancia de estas instancias para la mejora de la convivencia democrática dentro de la escuela, como lo han indicado reconocidos investigadores (Feito, 2011; Fernández-Enguita, 1992).

El otro contexto de estudio corresponde a España, en donde, a nivel de gobierno central, se han establecido algunos principios respecto al acceso a los cargos directivos y las atribuciones de los Consejos Escolares, los cuales están conformados desde la década de los años 80. En específico, el Consejo Escolar tiene competencias y obligaciones determinadas como la aprobación y evaluación de las normas, la programación anual del centro -esto sin perjuicio de las competencias propias del Claustro de profesores-y también tiene injerencia en las elecciones de los directivos; a su vez, debe decidir respecto al ingreso de estudiantes según la normativa vigente; conocer y velar por la adecuada resolución de conflictos y convivencia en el centro; aprobar la obtención de recursos para instalaciones y equipo escolar; fijar directrices sobre colaboraciones del centro con organismos administrativos y otros centros; analizar y valorar el funcionamiento del centro y del rendimiento escolar; elaborar propuestas e informes sobre el centro y otras que la administración indique, como queda determinado en Ley Orgánica 
de Educación 2/2006, de 3 de mayo.

En la Comunidad de Cataluña, donde se enmarca el estudio, se presenta como característica fundamental el uso de la lengua catalana como vía de la enseñanza, además de la celebración de fiestas tradicionales y transmisión de una cultura ancestral valorada por el pueblo catalán. En lo que respecta a la formación de directivos, se ha establecido una tendencia al fortalecimiento del director a través del perfeccionamiento del profesorado en liderazgo pedagógico, dirección estratégica y desarrollo de responsabilidades, a fin de que puedan adquirir habilidades para llegar a ser directivos en los centros públicos. De la misma manera, se han impulsado jornadas de reflexión y formación del profesorado y directivos en ejercicio sobre buenas prácticas en gestión, en el marco del Proyecto de Calidad y Mejora Continua del Departamento de Enseñanza de la Generalidad de Cataluña, que se desarrolla desde el año 1999. Todo lo anterior, se suma a la formación permanente de directivos y del personal administrativo sobre temáticas de gestión enfocada a la excelencia, como se indica desde el Departamento de Enseñanza de esta Comunidad Autónoma.

Ante este panorama, como se ha mencionado, se presentan los análisis de las visiones de dos directivos de escuelas secundarias en Chile y dos de España, quienes han buscado que, en sus institutos, se fomente la vida democrática a través de diversas prácticas. El foco de interés en las entrevistas fue la experiencia de participación y democracia que promovían los directivos desde sus roles para y con el estudiantado. El análisis y la caracterización de estas perspectivas, permitió identificar aspectos que fomentan una mejora en la vida democrática al interior de los institutos y facilitan la reflexión a quienes están inmersos en estos roles.

\section{Método}

Las entrevistas realizadas se enmarcan en un estudio de casos comparado entre cuatro institutos de Enseñanza Media, como se denominan en Chile, o Secundaria, nominación empleada en España y otros países. Los cuatro institutos se seleccionaron por haber sido reconocidos, dentro de sus comunidades, por su esfuerzo en la promoción de la participación de los estudiantes. Tanto en Chile como en España, este reconocimiento fue identificado a través de la revisión de los sitios web de municipalidades y periódicos de la zona, donde eran mencionados los institutos ante sus recurrentes actividades de compromiso cívico como seminarios, colaboración con otras escuelas y organizaciones de voluntariado.

Tras la selección y aprobación de la investigación por parte del instituto, se dio inicio a la fase de toma de datos, proceso en que los cuatro institutos escogidos fueron abordados en profundidad durante un periodo de tres meses. En este período, se conocieron y comprendieron las dinámicas de los directores de los centros, los cuales fueron entrevistados en dos momentos: primero, a través de una conversación abierta y luego, por medio de una entrevista en profundidad, de tipo cerrada. Los datos obtenidos en la entrevista en profundidad, se analizaron 
desde la perspectiva de la teoría fundamentada, por medio del uso del software ATLAS.ti, lo cual permitió categorizar, según la emergencia de tópicos desde los propios entrevistados. Los objetivos planteados eran comprender la perspectiva de los directivos respecto a su rol en la facilitación y promoción de la participación de los estudiantes y la formación democrática; en consecuencia, a continuación, no se expresan comparaciones entre directivos, sino que se describen las categorías y sus principales énfasis, a fin de conocer más profundamente el pensamiento de los líderes de institutos que han demostrado tener interés en el desarrollo de mejores aprendizajes en formación ciudadana, para participar de manera democrática.

\section{Resultados}

Respecto a las categorías que reflejaron el pensamiento directivo sobre la gestión y la promoción de la participación de los estudiantes, a lo largo de las entrevistas, se presentaron variados ámbitos. Las ideas principales fueron agrupadas, según la saturación demostrada, en tres grandes áreas como se describe a continuación.

\section{Convicciones personales}

En primer lugar, al analizar las entrevistas realizadas a los cuatro directivos, se encontraron énfasis temáticos, particularmente en lo referido a los fines de las prácticas que promovían ellos mismos y que, en definitiva, habían llevado a que se desarrollen en todo el instituto. Estos fines se relacionaron especialmente en una categoría amplia, denominada "Convicciones personales", que surgió de la reiterada visión presentada por los directivos respecto a que, tanto sus acciones diarias como los proyectos que promovían en los institutos, nacían motivados por ideas personales. Esta categoría agrupó conceptos e ideas de la vida en la escuela, a las que los directivos eran profundamente fieles. Por ejemplo, varios indicaron que su propia experiencia juvenil estuvo vinculada con actividades de tipo responsabilidad social con sus barrios o sectores más empobrecidos de la sociedad. En consecuencia, los directivos indicaron que la creación de espacios para que los jóvenes decidieran y participaran en distintos tipos de actividades, que no se relacionaban con asuntos académicos, era un aporte a su formación integral porque, principalmente, creían que la educación no formal era un aspecto transformador y buscaban promoverlo. Tal como se indica a continuación:

Para mí la participación de los jóvenes en la educación forma parte así, de lo que se llama así técnicamente educación integral. Porque si nosotros conseguimos que los alumnos participen, por ejemplo en las actividades del centro estamos haciendo un crecimiento, a los chavales yo creo que a nivel competencial y si aparte conseguimos que esta participación sea en las entidades o asociaciones del barrio, lo que estamos consiguiendo es abrirle puertas, que a lo mejor tardarían en conocer y de esta forma ya las conocen con más facilidad con lo cual se produce un crecimiento como persona, paralela al enseñamiento. (75:75 Directivo 1, 
Instituto 1, España)

Desde esa mirada, indicaron que los jóvenes debían hacerse cargo de determinados problemas de su entorno, ayudar a las organizaciones que lo necesitan y que, para eso, debían tomar decisiones, buscar soluciones a problemas y luego, plantearlos a los adultos del centro. También fueron enfáticos en indicar que la participación de los jóvenes en las diversas actividades que se promovían a nivel de institución u organizaban como directivos, era un espacio para potenciar las capacidades de los estudiantes en áreas no académicas, a modo de beneficiar su desarrollo integral. Como se observa a continuación:

Porque tal como plantea nuestro lema de 'formar líderes para el día de mañana', mi intención es que ese líder se inserte en la sociedad o en el sector, en la sociedad en general y ahí sea capaz de transformar el medio donde está. Esa es nuestra intención, esa es una de nuestras misiones como colegio, por eso me interesa que vean la sociedad, que la conozcan, que la vivan en carne propia, que vivan lo que sucede. (24:24 Directivo 1, Instituto 1, Chile)

En esta categoría, también destacó la emergencia de un tipo de liderazgo focalizado en la integración de los estudiantes a su comunidad y el desarrollo de la responsabilidad social, como parte de la convicción de que los estudiantes podían ser líderes de sus localidades en el futuro. Los directivos expresaban un alto valor al desarrollo de capacidades en los estudiantes por medio de las experiencias educativas en el mundo real y con los diversos grupos que componían los barrios próximos al Instituto. Los cuatro directivos entrevistados manifestaron confiar en el positivo impacto que tendría, para la formación de los jóvenes, participar en diferentes actividades a lo largo de su experiencia escolar.

\section{Diálogo y buena convivencia}

Otro aspecto destacado por los directivos y que, sin lugar a dudas, es un elemento de desarrollo y aprendizaje de la vida en democracia desde la escuela, correspondió a la importancia dada al diálogo y la buena relación de convivencia entre todos los integrantes de la comunidad escolar. En particular, uno de los directivos manifestó un alto desarrollo de técnicas de negociación y diálogo con los estudiantes, a fin de que ellos pudiesen resolver sus problemas por medio de la conversación con todos los miembros de la comunidad; es decir, padres, docentes y los mismos directivos.

Así también, destacó la búsqueda de la autonomía de los estudiantes a través del desarrollo de actitudes de responsabilidad frente a los asuntos propios del instituto y, principalmente, hacia los "permisos" que se obtenían con los directivos. Otros directivos señalaron mantener políticas de puertas abiertas en el Instituto, para que cada joven pudiese acceder a conversar con él mismo o con quien lo necesitara cuando hiciera falta; sin embargo, esta información no estaba explicitada en algún documento del centro, sino que permanecía en los niveles informales. Del mismo modo, 
otros se refirieron al diálogo y consenso desde una perspectiva teórica, más que a acciones cotidianas o relacionadas con espacios formales, como se observa en las siguientes citas:

Al final las tiene que tomar una persona (las decisiones) pero si tú consigues que la gente participe, que la gente vaya entendiendo, siempre es mucho más fácil a tomar, más fácil no, es más difícil porque hacer que todo el mundo pensemos es más difícil, pero las decisiones que se toman consensuadas creo que están mucho más decididas, o sea que te las tomas mucho más tuya que no las cosas que vienen impuestas. (92:92, Directivo 1, Instituto 2, España)

Los niños nuestros tienen todas las facilidades para acceder a los distintos niveles del colegio, aquí no están las puertas cerradas, ellos pueden venir, conversar conmigo el día que quieran o concertar entrevistas, pedir reuniones y así con los distintos directivos, las unidades del colegio, en eso nosotros tenemos una filosofía y es permanente de que ellos no sientan de que hay una barrera que nos separa, eso es así y lo puedes constatar tú porque ha sido históricamente así. (45:45 Directivo 1, Instituto 2, Chile)

Particularmente interesante, fue la visión de uno de los directivos que estimaba que el diálogo debía fomentarse desde las prácticas, para lo cual promovía planes de mediación de conflictos entre estudiantes que demandaban un compromiso de toda la comunidad, con el propósito de que fueran eficaces en su puesta en práctica. A su vez, él mismo se encontraba diseñando, junto con otros docentes, una asignatura de promoción de la participación que permitiera a los estudiantes dialogar sobre los asuntos de su interés, tal como se alcanza a comprender en esta descripción:

De momento estamos acabando de perfilar bien la asignatura y loúnico que estamos haciendo es de todo lo que hay en el centro para hacer difusión, para que se entere todo el alumnado de lo que hay, de donde puede participar y de donde puede presentar propuestas de mejora. Esta es un poco la idea mía, es decir, dicho de otra manera, que los alumnos sean conscientes de todo lo que hay en el centro para poder utilizarlo como recurso para ellos mismos, pero que a más y más tengan voz para cambiar, modificar o proponer todo tipo de actividades, desde la participación del alumnado en la sesión de evaluación hasta la participación de los alumnados en actividades extraescolares o actividades del barrio. (85:85, Directivo 1, Instituto 1, España)

De este modo, los líderes de los institutos involucrados expresaron una alta valoración al diálogo con los estudiantes como una vía de resolución de problemas, pero además, una forma de convivir y desarrollar capacidades en los estudiantes.

\section{Ejercicio del rol directivo}

Otra categoría que surgió de las entrevistas, fue la visión de los alcances de su rol que manifestaron tener los directivos, pues si bien había diversidad, existía un claro énfasis en que el 
ejercicio del rol pedagógico de sus cargos se vinculaba, principalmente, a la convivencia escolar y no a los aspectos curriculares de cada asignatura.

De este modo, predominó en los entrevistados la visión de que los docentes son los encargados de velar por el qué y cómo de los aprendizajes dentro del aula, en tanto ese ámbito correspondía a la autonomía profesional docente, y todos coincidieron en que debía ser respetado, como se puede leer en las frases siguientes:

Yo creo que al interior del aula es donde a lo mejor pudiesen darse mayores complicaciones, porque claro al interior del aula es como un concepto más bien estructurado, es muy estructurado donde el profesor tiene la responsabilidad sobre ciertos contenidos y ciertas competencias que tiene que trabajar y que tiene que desarrollar, por lo cual, creo que en el interior del aula la participación del alumno no es tan significativa. (18:18 Directivo 1, Instituto 1, Chile)

En cuanto a las materias ahí ya es un poco más personal de cada profesor, por ejemplo, hay profesores que la asignatura la dan a nivel de trabajo cooperativo con lo cual la participación de los alumnos es bastante elevada, mientras que hay profesores que trabajan a nivel de clase magistral, con lo cual la participación del alumnado es bastante baja. (87:87 Directivo 1, Instituto 1, España)

Sin embargo, pese a la contundencia de las afirmaciones respecto al tema, sí se apreció una diferencia entre los directivos, dado que solo dos se implicaban activamente en las actividades que los jóvenes realizaban fuera de los ámbitos académicos, porque consideraban que era parte de su rol colaborar en dar forma a las ideas de los estudiantes y apoyarlos en el desarrollo de las actividades derivadas. Los otros directivos indicaron que los jóvenes debían avanzar hacia aquello que les interesaba y que el instituto promovía, pero no se involucraban personalmente, tal y como se observa en esta cita: Ellos buscan junto con su profesor lo que quieren hacer y una vez que lo definen lo conversamos, le vamos como dándole más forma, más cuerpo y respaldando por supuesto (11:11 Directivo 1, Instituto 1, Chile).

Esta diferencia reveló una percepción distinta sobre el ejercicio del liderazgo educativo, ya que para algunos existía el interés de ejercer un rol pedagógico activo desde la propia participación, más que aprobación o rechazo de las iniciativas que los jóvenes pudiesen plantear sobre asuntos no académicos.

\section{Discusión}

A la luz del análisis realizado, se identificó una clara tendencia de los directivos de los institutos seleccionados para promover prácticas democráticas en la línea del diálogo y formación en compromiso ciudadano, en tanto los líderes de estos institutos realizaban diversas acciones motivados, esencialmente, por una convicción personal, lo cual situaría dentro de los aspectos morales del ejercicio de sus cargos, la formación democrática y ciudadana del alumnado. Elemento que se relaciona, estrechamente, con la visión de democracia descrita en el desarrollo teórico, en 
la cual todos los ciudadanos han de ser corresponsables de los asuntos que les afectan, como es la educación en habilidades y valores para participar de la vida en sociedad (Cogan \& Derricott, 2000).

Este aspecto pone en relieve la necesidad de visualizar, políticamente, la importancia que tiene la formación democrática y para participar en la sociedad actual, en tanto el líder de la escuela es quien puede fortalecer estos aspectos, a través de la comunicación adecuada a los demás integrantes de la comunidad escolar y la propia distribución de funciones, a fin de que no se personalice una tarea de carácter colectivo (Harris, 2012), características que favorecería una experiencia democrática para los jóvenes y los mismos profesores.

Por cuanto, como se observó en los análisis, existe en los directivos entrevistados una clara visión de que su rol permitía impulsar la participación de los estudiantes en pro de su formación democrática; sin embargo, este rol era limitado y se mantenía al margen de la vida académica, porque comprendían que ese espacio correspondía a los docentes. De este modo, se percibió la ausencia de relatos en torno a experiencias educativas que podrían comprenderse dentro de lo formal, como las propias dinámicas de aula, la resolución de conflictos por asuntos académicos, entre otros.

Sin duda, lo anterior es de importante consideración, si se estima que se ha identificado que los propios docentes valoran, altamente, el trabajo colectivo que permita mejorar los aprendizajes en distintas áreas y que además, favorezca las relaciones de confianza, respeto y empatía entre miembros de la comunidad educativa, como lo han indicado Coronel (2005) y Tapia-Gutiérrez et ál. (2011). En consecuencia, esta situación, a la luz de los resultados, podría indicar que los directivos que participaron de este estudio y demostraron tener un alto interés por el diálogo y estrategias de mediación con los estudiantes, podrían acceder al espacio académico y ejercer una mayor influencia en el sentido de promover instancias dialógicas.

La no reflexión sobre los alcances que establecen estas diferencias y desencuentros entre las expectativas sobre el trabajo que desempeñan directivos y docentes en los establecimientos educaciones de secundaria, compromete indirectamente la formación democrática de los estudiantes, en tanto el diálogo entre docentes y directivos sobre esta temática, permitiría aunar criterios sobre cómo fomentar el trabajo en equipo y el desarrollo de aprendizajes sobre temas de formación democrática como el diálogo y la convivencia. En consecuencia, que quienes ejercen distintos grados de responsabilidad en la formación de los jóvenes, establezcan acuerdos sobre estos asuntos, pues permitiría que las visiones de los directivos traspasen el ámbito de las convicciones personales y se sitúen en la impronta de la escuela y por último, en los espacios de desarrollo profesional de los docentes.

El impacto en la cultura escolar de estos tópicos, es particularmente importante si se comprende que la democracia situada desde la práctica favorece que, en la escuela, el ejercicio de autoridad parezca justo y legítimo a quienes lo soportan, tanto alumnos como docentes. Característica que colabora en generar mejores ambientes de aprendizaje y convivencia escolar (Dubet, 2006). De este modo, vivir valores democráticos en la experiencia escolar es un ámbito 
de aprendizaje potencial que trasciende desde el Proyecto Educativo de cada centro escolar y en la generación de un desarrollo de la responsabilidad social, la resolución de conflictos en el día a día y a la propia vida del aula. Aspecto que, por su envergadura, involucra a toda la comunidad escolar y niveles educativos, como lo han presentado en su compilación de experiencias democráticas, mencionada anteriormente con los autores Puig, Martín y Trilla (1998).

Por otra parte, si bien es compartida la visión de que el éxito en el aprendizaje en estas y otras materias no requiere de un único modelo de acciones ni de liderazgo (Hernández-Castillo, Murillo \& Martínez- Garrido, 2014), sí se estima relevante que los propios líderes de las escuelas actuales revisen su quehacer desde la óptica de compartir enfoques para promover el diálogo y la búsqueda de caminos comunes en la formación democrática dentro de sus escuelas. Lo cual, demandaría inminentemente la distribución de responsabilidades en los asuntos de formación pedagógica como son la educación democrática y para participar como ciudadano, más allá de la importantísima consideración moral individual de cada quien ejerza un cargo. Sin duda, los estudiantes aprenden en la propia experiencia escolar y eso involucra la observación de cómo se organiza su entorno, así la responsabilidad en la eficacia de estos aprendizajes depende, en buena medida, de que se considere a los propios docentes, apoderados y personal administrativo de la escuela, como corresponsables del logro de aprendizajes y se les facilite la aceptación de desafíos profesionales en estas materias.

\section{Conclusiones}

A partir de la información obtenida y analizada a lo largo de este estudio, se estima necesario establecer lineamientos y orientaciones que colaboren a los directivos en el propio desarrollo de su actividad profesional, como en la generación de comunidades docentes donde se compartan las decisiones, se distribuyan las responsabilidades y compartan visiones sobre la educación. A fin de que estas estructuras acompañen a los estudiantes en un desarrollo de la vida en democracia más a conciencia y eminentemente práctico, como los mismos directivos han indicado necesario, desde sus propias convicciones, y como se ha demostrado ser necesario para las democracias actuales (Morin, 2011).

A su vez, se muestra la necesidad de avanzar en orientaciones para que las escuelas, sin aplicar recetas, sino a través del análisis de sus propios contextos, puedan generar directrices que aporten a una cultura escolar inclusiva y democrática. Es decir, que incorpore las perspectivas de todos los actores de la comunidad escolar y construya con ellos, las transformaciones que se necesitan, pero considerando las diferencias entre grupos e individuos, además de que favorezca la disminución de barreras para el aprendizaje y participación de todos los estudiantes (Booth \& Ainscow, 2002). Desde décadas pasadas, destacados autores han indicado la relevancia de estos puntos, especialmente porque colaboran en mejorar la cultura y convivencia al interior de la escuela y aportan a la consecución de aprendizajes de calidad. Fullan y Hargreaves (1999) lo indicaron con fuerza en la década de los 90: 
El director es un modelo de rol para el trabajo en equipo dentro y fuera de la escuela. Es interesante y es paradójico: los directores que comparten la autoridad y establecen condiciones propicias para la delegación, incrementan de hecho su influencia sobre las actividades de la escuela al mismo tiempo que trabajan con el personal en la producción de mejoras. (p. 152)

Desde esta perspectiva, entonces, vale la pena considerar los hallazgos mostrados por esta investigación, en tanto existe en los entrevistados una destacada valorización de la democracia como práctica educativa. Lo que facilita que, desde estas positivas condiciones, se pueda emprender hacia los caminos de la cooperación y distribución, a modo de impactar más ampliamente en la formación democrática de los estudiantes y por qué no, de los mismos docentes y directivos que se desarrollan profesionalmente en las escuelas.

Finalmente, a partir de la investigación realizada, es interesante destacar que se observa la necesidad de continuar realizando estudios sobre liderazgo y gestión educativa en países como los implicados en este caso, los cuales se encuentran impulsando importantes reformas a su sistema educativo. Especialmente en Chile, donde el proceso actual de reforma educativa generará transformaciones en la organización educativa que, de seguro, impactará en los directivos y su relación con docentes y estudiantes. Es así, como se abren nuevos escenarios para avanzar hacia una cooperación entre los miembros de la comunidad educativa para reaccionar ante el cambio y construir, a lo largo de la experiencia educativa, espacios más democráticos.

\section{Referencias}

Arnstein, Sh. (1969). A ladder of Citizen Participation. Journal of the American Institute of planners, 35(4), 216- 224. Recuperado de: http://lithgow-schmidt.dk/sherry-arnstein/ladder-ofcitizen-participation.html

Beck, U. (2008). La sociedad del riesgo mundial: en busca de la seguridad perdida. Barcelona: Paidós.

Beetham, D. y Boyle, K. (1996). Cuestiones sobre la democracia, conceptos, elementos y principios básicos. Madrid: Los libros de la Catarata.

Bellei, C., Contreras, D. y Valenzuela, J. (eds). (2009). Ecos de la Revolución Pingüina. Avances, debates y silencios en la reforma educacional. Santiago, Chile: UNICEF-CEAE.

Bisquerra, R. (coord.) (2004). Metodología de la investigación educativa. Madrid: La Muralla.

Bolívar, A., López, J. \& Murillo, F. (2013). Liderazgo en las instituciones educativas. Una revisión 
de líneas de investigación. Revista Fuentes, 14, 15-60. Recuperado de: http://institucional. us.es/fuentes/numeros anteriores/firma anterior.php?vid=14

Booth, T. \& Ainscow, M. (2002). Índice de inclusión. Desarrollando el aprendizaje y la participación en las escuelas Unesco-CSIE. Recuperado de: http://www.eenet.org.uk/resources/docs/ Index\%20Spanish\%20South\%20America\%20.pdf

Camps, V. (ed). (2010). Democracia sin ciudadanos, la construcción de la ciudadanía en las democracias liberales. Madrid: Trotta.

Cogan, J. \& Derricott, R. (2000). Citizenship for the 21st century. An international perspective of education. Wales: Kogan Page.

Coronel, J. (2005). El liderazgo del profesorado en las organizaciones educativas: temáticas para su análisis e investigación. Revista Española de Pedagogía, 232, 471-490. Recuperado de: file:///C:/Users/equipo\%201/Downloads/Dialnet-ElLiderazgoDelProfesoradoEnLasOrgani zacionesEducat-1373230.pdf

Chomsky, N. (2010). La democracia de mercado en el sistema neoliberal: realidad y doctrina. La (des) educación. Barcelona: Crítica.

Dubet, F. (2006). La Escuela de las oportunidades ¿Qué es una escuela justa? Barcelona: Gedisa.

Feito, R. (2011). Los retos de la participación escolar. Madrid: Morata.

Fernández- Enguita, M. (1992). Poder y participación en el sistema educativo: sobre las contradicciones del sistema escolar en un contexto democrático. Barcelona: Paidós.

Fullan, M. \& Hargreaves, A. (1999). La escuela que queremos. Los cambios por los que vale la pena luchar. Buenos Aires: Amorrortú.

Fullan, M. (2001). Leading a culture of change. USA: Jossey- Bass.

Goleman, D. (2002). El líder resonante crea más. Barcelona: Plaza \& Yañez.

Habermas, J. (1999). La inclusión del otro. Barcelona: Paidós.

Habermas, J. (2000). La constelación posnacional: ensayos políticos. Barcelona: Paidós.

Harris, A. (2012). Liderazgo y desarrollo de capacidades en la escuela. Santiago: Fundación Chile.

Harris, A. \& Chapman, C. (2002). Democratic leadership for school improvement in challenging 
contexts. International Electronic Journal for Leadership in Learning, 6(9). Re c u p e r a do de: http://eprints.gla.ac.uk/75099/

Held, D. (1997). La democracia y el orden global, del estado moderno al gobierno cosmopolita. Barcelona: Paidós.

Hernández-Castillo, R., Murillo, F. \& Martínez-Garrido, C. (2014). Factores de ineficacia escolar. Revista Iberoamericana sobre Calidad, Eficacia y Cambio en Educación, 12(1), 103-118. Recuperado de: http://www.rinace.net/reice/numeros/vol12num1.htm

Klein, N. (2007). The shock doctrine. The rise of disaster capitalism. New York: Picador.

Lorenzo, D. (2005). El liderazgo en las organizaciones educativas: revisión y perspectivas actuales. Revista Española de Pedagogía, 63(232),367-388. Recuperado de:file:///C:/Users/equipo\%201/ Downloads/Dialnet-ElLiderazgoEnLasOrganizacionesEducativas-1373221.pdf

Morin, E. (2011). La vía. Para el futuro de la humanidad. Barcelona: Paidós.

Muñoz, G. (2011). Representación simbólica de los consejos escolares como estrategia para democratizar la cultura escolar: una lectura interpretativa desde la voz de los sujetos sociales. Estudios pedagógicos, 37(1), 35-52. Recuperado de: http://www.scielo.cl/scielo. php?script=sci arttext\&pid=S0718-07052011000100002\&lng=es\&tlng=es. $\quad 10.4067 /$ $\underline{\text { S0718-07052011000100002 }}$

Murillo, F. (2006). Una dirección escolar para el cambio: del liderazgo Transformacional al liderazgo Distribuido. Revista Electrónica Iberoamericana sobre Calidad, Eficacia y Cambio en Educación, 4(4e). Recuperado de: http://www.rinace.net/arts/vol4num4e/art2.htm

Navarro, L. (2007). Aseguramiento de la calidad de la gestión escolar: ¿De qué estamos hablando? Docencia, 31, 30-38. Recuperado de: http://www.revistadocencia.cl/pdf/20100731211150.pdf

Ley 20.370. General de Educación. Recuperado de http://www.leychile.cl/ Navegar?idNorma $=1005923$

Ley 19.979. Modifica el régimen de Jornada Escolar Completa Diurna y otros cuerpos

legales.

Recuperado de: http://www.leychile.cl/Navegar?idNorma=232146

Puig, J., Martín, T. \&Trilla, J. (1998). Cròniques pera una educación democrática. Vic: Eumo Editorial. Riggio, R., Chaleff, I. \& Lipman-Blumen, J. (eds). (2008). The Art offollowership. How Great Followers 
create Great Leaders and Organizations. USA: Jossey- Bass.

Riveros-Barrera, A. (2012). La distribución del liderazgo como estrategia de mejoramiento institucional. Educación y Educadores, 15(2), 289-301. Recuperado de: http://www.redalyc. org/articulo.oa?id=83424870008

Senge, P. (2005). La quinta disciplina: cómo impulsar el aprendizaje en la organización inteligente. Buenos Aires: Granica.

Senge,P.(2012).Creatingschoolsforthefuture, notthepastforallstudents.LeadertoLeader,2012(65), 44-49. Recuperado de: http://www.readcube.com/articles/10.1002\%2Fltl.20035?r3 referer $=$ wol\&tracking action $=$ preview click\&show checkout $=1 \&$ purchase referrer $=$ onlinelibrary.wiley.com\&purchase site license $=$ LICENSE DENIED

Shulz, W., Ainley, J., Fraillon, J., Kerr, D. \& Losito, B. (2009). ICCS 2009 International Report: Civic knowledge, attitudes, and engagement among lowersecondary school students in $\quad 3 \quad 8$ countries. Amsterdam: IEA- MultiCopy Netherlands b.v.

Tapia-Gutiérrez, C., Becerra-Peña, S., Mansilla-Sepúlveda, J. y Saavedra-Muñoz, J. (2011). Liderazgo de los directivos docentes en contextos vulnerables. Educación y Educadores, 14(2), 389-409. Recuperado de: http://www.redalyc.org/articulo.oa?id=83421404010

Touraine, A. (2005). Un nuevo paradigma para comprender el mundo de hoy. Barcelona: Paidós. 